\title{
September/October 2011
}

\section{SurfNet-Technology of the Future-Today!}

Vol 2, Issue 5

\section{Supporting Release Integration Decisions with Collaborative Team Tools}

\section{By Shaun Phillips and Jonathan Sillito}

Managing the release of a software product is difficult, especially when the software is developed by different teams working in parallel. Combining or integrating the work of multiple development teams has to be planned carefully to avoid delaying a release or releasing a low quality product. Decisions have to be made about when different pieces of work should be integrated, how much time and effort will be required, and which tests should be run to catch any defects introduced during integration.

Our research aims to support these collaborative decisions with software tools. In a typical scenario, a release meeting room is equipped with a digital surface that displays information about ongoing parallel work and warns of any potential problems. As depicted in the tool mock-up, realtime branch health is visualized based on a variety of metrics (open bugs, test results, unmerged and conflicting code, etc.). The meeting attendants can then manipulate the surface to quickly find

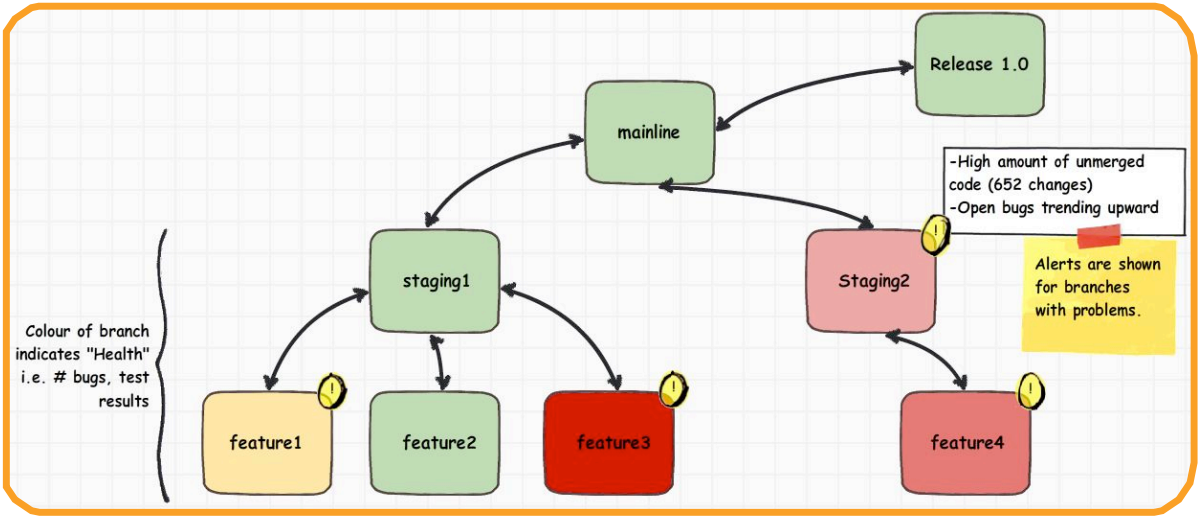

A collaborative tool used to solve complex integration problems (design mock-up)

relevant information and address the issues.

These tools are currently being developed in partnership with a large Canadian software company.

Essentially, the tools will facilitate finding problems sooner in parallel development environments. By identifying integration problems before they occur, and fostering cross-team collaboration to solve integration problems, the tools will promote greater predictability and efficiency in the software release process.

If you would like more information, or you would like to be involved in our research, please contact phillist@ucalgary.ca.

This research is one of SurfNet's special projects. To find out more about this research project, please visit the SurfNet website http:// www.nsercsurfnet.ca/pmwiki.php? $\underline{\mathrm{n}=\text { SurfNet.SillitoPulse. }}$

\section{In This Issue}

- Supporting Release Integration Decisions with Collaborative Team Tools

- Digital Tabletops and the Western Canadian Farmer

THANK YOU

to everyone who attended the Summer 2011 Workshop and Industry Open House. It was a huge success!

- Research In Review 


\section{Digital Tabletops and the Western Canadian Farmer}

\section{By Teddy Seyed}

Decisive Farming is an Alberta based company that offers highly modern farming approaches and services to Western Canadian farmers. These services include soil fertility analysis, risk management and precision farming.

The process of precision farming offered by Decision Farming is extremely advanced, and involves the combination of several sources of data. This includes data such as soil analysis results, satellite imagery, crop pricing and weather information. Currently, this process is done in a paper-based approach and includes numerous maps and spreadsheets. For a farmer,

visualizing and understanding this data can be somewhat complicated.

The Agile Software Engineering Lab at the University of Calgary in collaboration with Decisive Farming is working to modernize their cutting-edge process and streamline it into a highly visual and interactive experience.

The approach we have taken

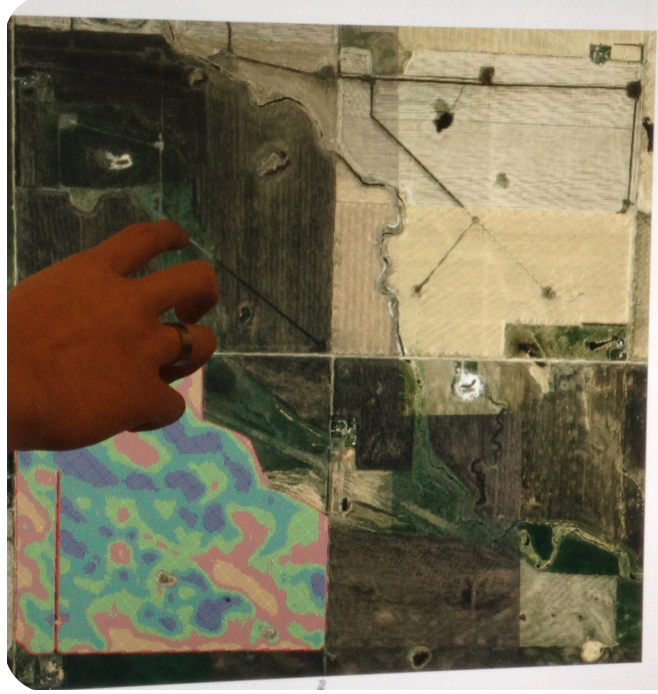

replaces the paper-based maps and documents and uses a digital tabletop as a medium for interaction. Farmers are able to collaboratively sit through the process of precision farming and visualize their data much easier. For Decisive Farming, the benefits of this approach are numerous as the entire process is now interactive and

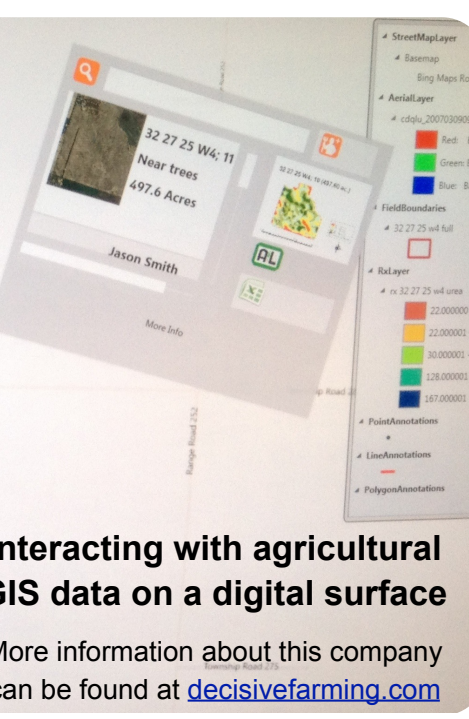

streamlines several of their current paper-based techniques.

In the future, we plan to create an even more innovative visualization, utilizing multiple surfaces, with surfaces dedicated to specific types of data. This will allow for a more immersive and complete experience and minimize information overload for farmers.

\section{Research in Review}

For SurfNet contact information please go to: www.nsercsurfnet.ca/pmwiki.php?n=SurfNet.Contact

\section{Summer 2011 SurfNet Workshop and Industry Open House a Huge Success}

The University of Calgary once again played host to our annual workshop. Added to last year's three days of exciting talks, demos, dialogs and social networking, were tutorial sessions and tours. Our students did a fantastic job teaching each other all they know in the full day of tutorial sessions; and both SMART Technologies and the Taylor Family Digital Library provided excellent tours. The workshop certainly resulted in many collaboration opportunities and fantastic team building experiences.

\section{New Special Projects}

Thank you to all students who submitted project proposals. The following seven projects were selected for funding:

- Meng-Wei Chang: "Surface-Based Text Analysis Through Descriptive Rendering"

- Túlio de Souza Alcantara: "Using Machine Learning to Recognize Gestures"

- Stevenson Gossage: "A Distributed Agile Card Wall"

- Christian Grossauer, Florian Perteneder: "Sketch-based Mathematics in Education"

- Theodore D. Hellmann: "Automated and Rule-Based Exploratory Testing of Surface-Based Applications"

- Hammad Ullah Khan: "Table-based Real-time Monitoring of Distributed Virtual Worlds"

- Rojin S. Vishkaie: "Develop and Evaluate Best Practices for Interacting with GIS on Large Multi-Touch Displays"

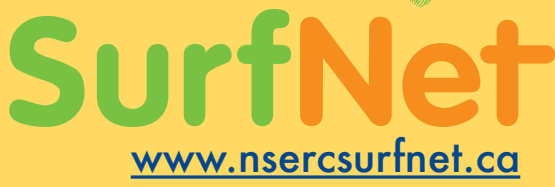

